\title{
An Interactive Online Educational Applet for Multiple Frequencies of Radar Observations
}

\author{
Ethan L. Nelson, Tristan S. l'Ecuyer, Adele L. Igel, and Susan C. van den Heever
}

$\mathrm{T}$ he use of active remote sensing is integral to accurate short-term weather forecasting and improving our understanding of precipitation processes in Earth's weather and climate system (National Academies of Sciences, Engineering, and Medicine 2018). In particular, utilizing radars that operate at shorter microwave wavelengths providing observations of multiple aspects of the atmosphere is crucial to systematic, process-level studies (e.g., Stephens et al. 2018). At the University of Wisconsin-Madison, a need for conveying the concepts underlying the importance of this multiple-frequency radar perspective in satellite remote sensing was identified in undergraduate atmospheric science courses. To that end, an online learning applet (or module) has been developed as a virtual laboratory tool to guide students through this important concept.

Remote sensing has indeed progressed to the point that multiple-frequency active instrument satellites [e.g., Global Precipitation Measurement Core Observatory (GPM-CO; Hou et al. 2014), Earthcare

AfFiliations: Nelson-Jet Propulsion Laboratory, California Institute of Technology, Pasadena, California, and Department of Atmospheric and Oceanic Sciences, University of Wisconsin-Madison, Madison, Wisconsin; L'ECUYER-Department of Atmospheric and Oceanic Sciences, University of WisconsinMadison, Madison, Wisconsin; IGeL-Department of Land, Air, and Water Resources, University of California, Davis, Davis, California; van den HeEver-Department of Atmospheric Science, Colorado State University, Fort Collins, Colorado CORRESPONDING AUTHOR: Ethan L. Nelson, enelson@jpl.nasa.gov

The abstract for this article can be found in this issue, following the table of contents.

DOI:10.II75/BAMS-D-I8-0249.I

A supplement to this article is available online (I0.II75/BAMS-D-18-0249.2)

(C2019 American Meteorological Society

For information regarding reuse of this content and general

copyright information, consult the AMS Copyright Policy.
(Illingworth et al. 2015)] and satellite constellations containing multiple active instruments [the Afternoon Constellation or A-Train (L'Ecuyer and Jiang 2010)] are being launched and actively collecting data. The use and design of these instruments are not without their own challenges, however. Since satellite radars operate at higher frequencies (shorter wavelengths) than conventional ground-based radars, they are more prone to the effects of attenuation and require an understanding of more complex radiative interactions, namely Mie scattering. Additionally, satellite instruments of any sort are limited by size and power given the requirements of spacecraft propulsion, instrument operation, and data communication. That means an educational tool on this subject should convey information not just about the synergy of multifrequency active instruments but also the trade-offs between instrument sensitivity, sensor complexity, cost, platform, and effectiveness of the instrument in sensing different atmospheric phenomena.

The lack of current learning media that emphasize the importance of the use of multiple-frequency active remote sensing in weather and climate contexts motivates an educational tool documenting the topic of multifrequency instrument observations. Core requirements designated at the conception of this learning module design are listed in Table 1 . The module

TABLE I. List of requirements for the online module of this activity.

Requirements

I. Grounded in proposed or hypothetical Earthobserving missions

2. Multiple frequencies of radar observations

3. Adjustable sensitivity for a given frequency

4. Vertical and horizontal cross-sections available

5. Simultaneous "truth" hydrometeor information for observations 
is designed to convey the information in a framework that allows for exploration of multiple frequencies of active measurements with different configurations following realistic Earth observation missions.

MODEL AND SIMULATED OBSERVATION DATA. Central to this online applet is a realistic three-dimensional scene to be explored that covers a large spatial area of some weather phenomena. The midlatitude, or extratropical, cyclone is a weather feature that consists of cold and warm frontal boundaries with different types of associated precipitation (e.g., Bjerknes 1919). Extratropical cyclones impact many major population centers in the world, and their lifetime and spatial extent provide the opportunity to examine a diversity of weather regimes that exists in the real world. A high-resolution cloud-resolving model is the ideal source for input data that are necessary for the module. In this case, the Regional Atmospheric Modeling System (RAMS) is used (Cotton et al. 2003; Saleeby and Cotton 2005). The model data are of a time step from a case study of a midlatitude cyclone evolving over the continental United States in April 2009 with horizontal resolution of $3 \mathrm{~km}$ and variable vertical resolution (Igel et al. 2013). A single model time step is taken of the weather system while the low pressure is centered over Kansas, with the warm front emanating due east from the low center, a dryline extending south of the low, and a cold front sitting to the west of the low. RAMS supplies a three-dimensional grid of atmospheric state variables (e.g., temperature, pressure) as well as microphysical information for all hydrometeors, or types of cloud and precipitation particles (e.g., cloud droplets, ice crystals).

With the environment of the synthetic scene defined, the next step is to generate what a satellite instrument would sense when orbiting over the model scene. This is accomplished with an instrument simulator. A variety of instrument simulators exist with a wide degree of varying complexity regarding not only the detail of the electromagnetic radiation interactions but also the orbital characteristics of the satellite (Tanelli et al. 2011, Bodas-Salcedo et al. 2011, and others). The most complex simulators can represent phenomena such as multiple scattering, nonuniform beam filling, Doppler spectra, slant angle, and surface backscatter contamination. Fortunately, many of these complexities are not necessary to demonstrate the major differences in performance of radars at multiple frequencies. Furthermore, choosing a simple simulator that can provide a direct one-to-one conversion between the model hydrometeor information and simulated instrument observation allows users to diagnose direct relationships between hydrometeor fields and the radar observations. A simulator that provides this one-to-one conversion is the Quickbeam radar simulator (Haynes et al. 2007).

Quickbeam is configurable to provide simulated radar observations for the frequencies of interest in weather and climate studies, including S-, Ku-, $\mathrm{Ka}-$, and $\mathrm{W}$-band. Hydrometeor mixing ratio and number concentration for graupel, hail, ice crystals, aggregates, rain, drizzle, and cloud droplets are used in the simulator along with state variables of pressure, temperature, and water vapor at all vertical levels. Reflectivities are simulated as single columns with hydrometeor attenuation included but not multiple scattering. After completing Quickbeam simulations, all of the necessary data for the module are available.

MODULE GENERATION. The online module can be separated into two main components: generated images of the data and the web page with tools to navigate the data. Because the module is restricted to a single model time step with predefined orbit tracks, the range of atmospheric cross sections needed is limited and the images can be statically generated ahead of time with a reasonably small volume of storage space. Prerendering would not be as feasible in the case of a module that allowed students to draw an orbit path free-form (though options such as this are planned; see "Development Roadmap" below). Six satellite orbits in each of the zonal and meridional directions and eleven planar views in the horizontal are selected to highlight the variety of regimes present in the model time step (Fig. 1). The horizontal planar views are intended to provide an additional comparison between the satellite perspective and traditional radar perspectives that employ mostly horizontal scan strategies. Additionally, two of the starker views are chosen as specific cases that have single-click access on the module page.

Images are all generated with the Python matplotlib module (Hunter 2007) using typical two-dimensional plotting routines. Specifically, images are generated of hydrometeor contents grouped into liquid and ice categories and radar reflectivities at each band of "current mission" and "improved mission" sensitivities. "Current mission" sensitivities resemble the advertised capabilities of radar instruments commissioned by the National 


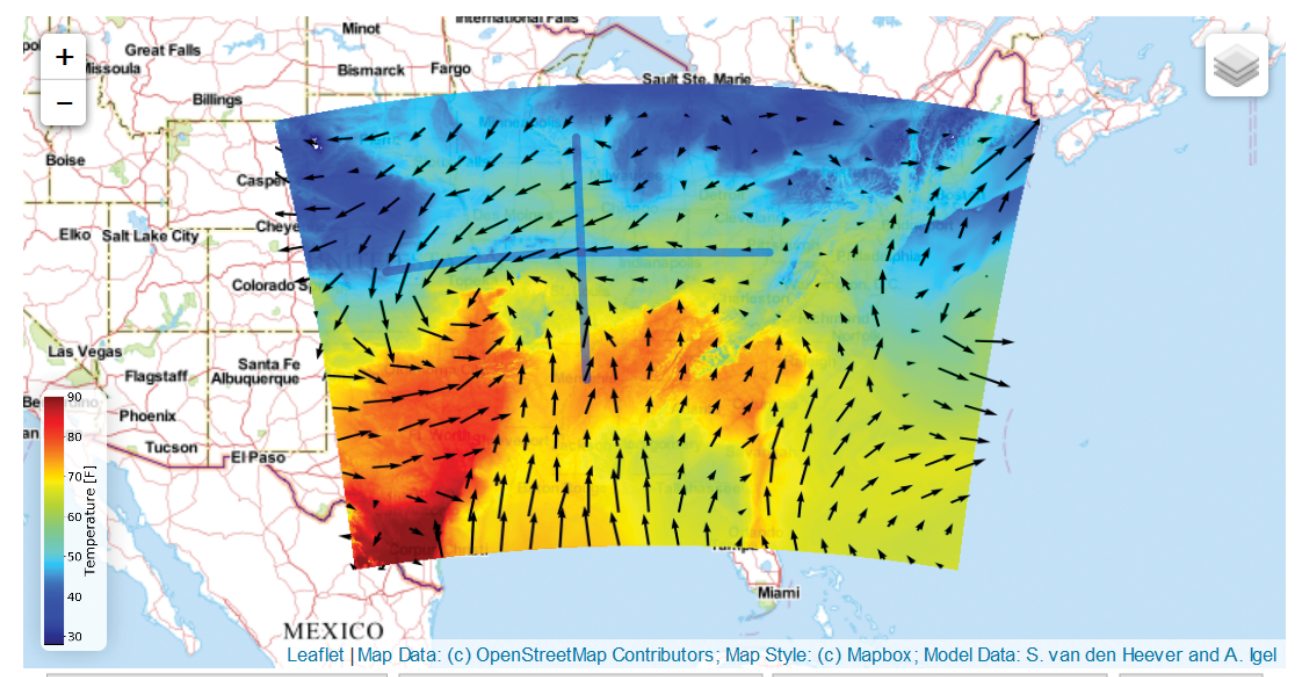

\begin{tabular}{|l|l|l|l|}
\hline Display Vertical Cross Sections Display Horizontal Cross Sections Improved mission MDR shown Attenuated \\
\hline
\end{tabular} Load Case \#1 Load Case \#2
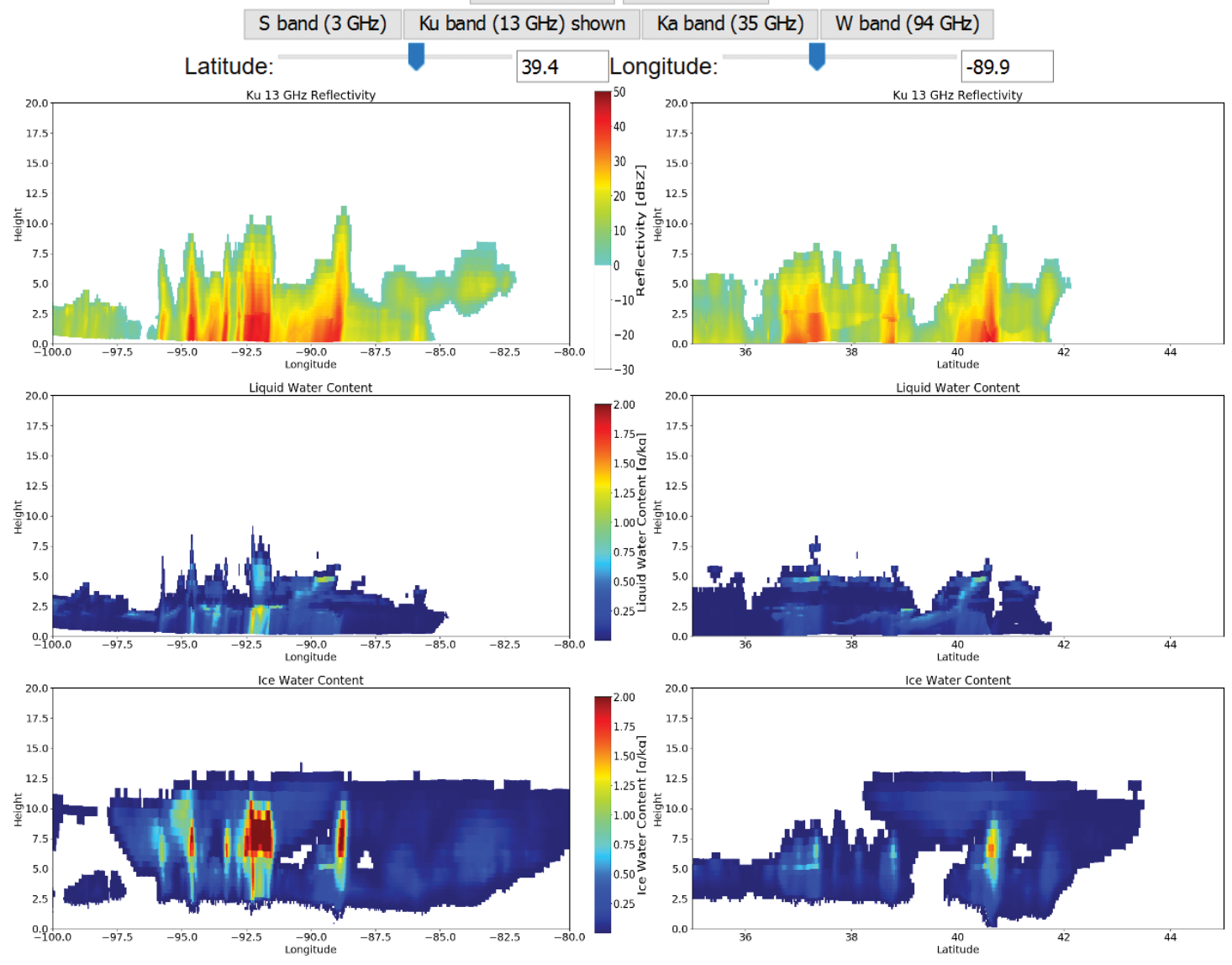

Cethan-nelson/radarlabviewer

Fig. I. A screen capture of the module displaying the layout of the model data and cross-section, including radar reflectivities and hydrometeor concentrations, as well as the navigation capabilities to load different instruments and cross-sections. 
Aeronautics and Space Administration [W-band: -30 dBZ, CloudSat (Tanelli et al. 2008); Ka-band: $12 \mathrm{dBZ}$, GPM-CO (Hou et al. 2014); Ku-band: $18 \mathrm{dBZ}$, Tropical Rainfall Measurement Mission and GPM-CO (Hou et al. 2014); S-band: $0 \mathrm{dBZ}$, National Aeronautics and Space Administration S-Band Dual Polarimetric Radar (Petersen and Wolff 2013)], while "improved mission" sensitivities are chosen as idealistic conceptions of future mission radars (W-band: - $30 \mathrm{dBZ}$; Ka-band: $0 \mathrm{dBZ}$; Ku-band: $0 \mathrm{dBZ}$; S-band: $0 \mathrm{dBZ}$ ) with grounding in the recently released Decadal Survey for Earth Observations from Space (National Academies of Sciences, Engineering, and Medicine 2018). Resolutions of the observations remain the same as the cloud-resolving model due to the radar simulator used, but this has the added benefit of one-to-one comparisons between the model state and observations as mentioned before.

The website content is written in Markdown and rendered with the Ruby Jekyll templating engine (https://jekyllrb.com), which generates a directory containing all of the site assets and rendered HTML pages. This engine is employed only as a convenience, and the site can be hosted without Jekyll, though the use of this engine provides the ability to host the site freely on GitHub (https://github.com). Manipulation of the site-meaning the exploration of images through frequency, cross section, and sensitivity selection-is controlled by basic JavaScript functionality. All images that were generated are stored in the same site repository for access through the website interface. Figure 1 is a browser screen capture of the web page displaying the layout with two crosssections selected.

LEARNING ACTIVITY. The main motivation behind this module development is for it to serve as a supplemental educational tool with the overarching learning goal to understand the differences in various radar frequencies and the usefulness of exploiting these differences in weather and climate research. There is an associated activity sheet (see online supplement) to guide students through the tool navigation and learning objectives that has previously been provided in three course offerings. The activity sheet contains a scaffolded open-ended set of questions that guides students through cases of certain weather regimes including convective cores with strong updrafts and ice contents, cirrus cloud anvil outflows with very small ice contents, and predominantly liquid cloud regions. For the final part of the activity, students are asked to design a satellite for optimal sensing of the full spectrum of clouds and precipitation with a monetary budget constraint and supplied costs of each sensor based on frequency and sensitivity. Physical concepts that are reinforced through the activity sheet include attenuation of high frequency signals in the presence of high hydrometeor contents, non-Rayleigh scattering of higher frequency signals, insensitivity of lower frequency instruments to smaller hydrometeors, and the information differences between top-down satellite instrument perspectives and horizontal planar ground radar scans.

DEVELOPMENT ROADMAP. The module has been included in three satellite and radar meteorology course offerings at the University of WisconsinMadison to date, either as an in-class laboratory activity or an out-of-class deeper thought assignment. An initial assessment of student learning gains and perceptions with the associated learning activity was completed through the University of Wisconsin-Madison Delta Program for Research, Teaching, and Learning Certificate Internship program. This assessment showed a positive reception and self-judged improvement in understanding from students. As part of the initial assessment, some aspects of the tool have undergone revision to improve the user experience and workflow based on informal student feedback. A formal learning assessment that can be disseminated is planned after undergoing human subjects board review.

We have identified five avenues of tool function expansion in the near future. The first is to implement a variable instrument sensitivity gauge instead of a binary switch to better diagnose the ability to sense different weather regimes. This will require either substantially more preprocessing and image generation or the use of in-browser rendering. The latter will likely increase the computer and browser requirements for users. The second avenue of expansion is to provide the ability for users to draw their own instrument orbit paths over the domain, which will also require in-browser rendering to accomplish. The third feature to add is variable footprint sizes by convolving single-column reflectivities from QuickBeam. This will enable more advanced learning topics of nonuniform beam filling, multiple scattering, and scan strategy. The fourth expansion is to include additional case studies for investigation. Doing so will allow for more focused exploration of 
certain atmospheric regimes, including wintertime frozen precipitation, tropical cyclones, and oceanic precipitation. The final planned feature is to provide built-in pricing estimates that will allow immediate insights into the science-cost tradespace that exists in mission formulation and planning. Pricing estimates can be added directly into the module, as opposed to existing only in the learning activity, either with the current applet state or in addition to any planned improvements.

SUMMARY. The topic of combining observations from instruments of different frequencies and sensitivities on remote sensing platforms has been underutilized in undergraduate and graduate atmospheric science curricula, but is vital to obtaining holistic views of the Earth climate system. An online education applet on this topic has been developed for use in radar and remote sensing atmospheric science courses that meets the requirements outlined in Table 1 . The developed applet attempts to reduce the curriculum gap by guiding students through atmospheric weather phenomena and allowing them to explore the performance of a variety of radar frequencies and characteristics.

This applet leverages a high-resolution cloud-resolving model and satellite radar simulator to provide a synthetic learning laboratory environment. It contains one-to-one comparisons between the simulated environment and approximate observations of that environment through the use of hypothetical remote sensing instruments. A limited set of orbits and planar views are selected to highlight advantages and shortfalls of potential instrument configurations. Future formal assessments of the learning tool will allows its effectiveness to be quantified and guide further improvement of the tool beyond currently planned development activities.

ACKNOWLEDGMENTS. The applet is hosted at http:// lecuyer.aos.wisc.edu/radarlabviewer/. The applet code is available at https://github.com/ethan-nelson/radarlabviewer and the atmospheric data are available at http://lecuyer.aos .wisc.edu/radarlabviewerdocs/. This work was supported in part by NASA Headquarters under the Earth and Space Science Fellowship \#NNX14AL35H. Learning activity development of this module occurred under the auspices of the University of Wisconsin Delta Program for Research, Teaching, and Learning, a project of the Center of the Integration of Research, Teaching, and Learning (NSF Grant \#0227592). The writing of this paper was supported by the
Jet Propulsion Laboratory, California Institute of Technology, under a contract with the National Aeronautics and Space Administration.

\section{FOR FURTHER READING}

Bjerknes, J., 1919: On the structure of moving cyclones. Mon. Wea. Rev., 47, 95-99, https://doi.org/10.1175/1520 -0493(1919)47<95:OTSOMC>2.0.CO;2.

Bodas-Salcedo, A., and Coauthors, 2011: COSP: Satellite simulation software for model assessment. Bull. Amer. Meteor. Soc., 92, 1023-1043, https://doi .org/10.1175/2011BAMS2856.1.

Cotton, W. R., and Coauthors, 2003: RAMS 2001: Current status and future directions. Meteor. Atmos. Phys., 82, 5-29, https://doi.org/10.1007/s00703-0010584-9.

Haynes, J. M., R. T. Marchand, Z. Luo, A. BodasSalcedo, and G. L. Stephens, 2007: A multipurpose radar simulation package: Quickbeam. Bull. Amer. Meteor. Soc., 88, 1723-1728, https://doi.org/10.1175 /BAMS-88-11-1723.

Hou, A. Y., and Coauthors, 2014: The Global Precipitation Measurement Mission. Bull. Amer. Meteor. Soc., 95, 701-722, https://doi.org/10.1175/BAMS -D-13-00164.1.

Hunter, J. D., 2007: Matplotlib: A 2D graphics environment. Comput. Sci. Eng., 9, 90-95, https://doi .org/10.1109/MCSE.2007.55.

Igel, A. L., S. C. van den Heever, C. M. Naud, S. M. Saleeby, and D. J. Posselt, 2013: Sensitivity of warmfrontal processes to cloud-nucleating aerosol concentrations. J. Atmos. Sci., 70, 1768-1783, https://doi .org/10.1175/JAS-D-12-0170.1.

Illingworth, A. J., and Coauthors, 2015: The EarthCARE Satellite: The next step forward in global measurements of clouds, aerosols, precipitation, and radiation. Bull. Amer. Meteor. Soc., 96, 13111332, https://doi.org/10.1175/BAMS-D-12-00227.1.

L'Ecuyer, T. S., and J. Jiang, 2010: Touring the atmosphere aboard the A-Train. Phys. Today, 63, 36.

National Academies of Sciences, Engineering, and Medicine, 2018: Thriving on Our Changing Planet: A Decadal Strategy for Earth Observation from Space. The National Academies Press, 716 pp., www.nap .edu/catalog/24938/thriving-on-our-changing -planet-a-decadal-strategy-for-earth.

Petersen, W. A., and D. B. Wolff, 2013: The NASA Polarimetric Radar (NPOL). NASA Technical Reports Server. 
Saleeby, S. M., and W. R. Cotton, 2005: A large-droplet mode and prognostic number concentration of cloud droplets in the Colorado State University Regional Atmospheric Modeling System (RAMS). Part II: Sensitivity to a Colorado winter snowfall event. J. Appl. Meteor., 44, 1912-1929, https://doi.org/10.1175 /JAM2312.1.

Stephens, G., D. Winker, J. Pelon, C. Trepte, D. Vane, C. Yuhas, T. L'Ecuyer, and M. Lebsock, 2018: CloudSat and CALIPSO within the A-Train: Ten years of actively observing the Earth system. Bull. Amer. Meteor. Soc., 99, 569-581, https://doi.org/10.1175 /BAMS-D-16-0324.1.
Tanelli, S., S. L. Durden, E. Im, K. S. Pak, D. G. Reinke, P. Partain, J. M. Haynes, and R. T. Marchand, 2008: CloudSat's Cloud Profiling Radar after two years in orbit: Performance, calibration, and processing. IEEE Trans. Geosci. Remote Sens., 46, 3560-3573, https://doi.org/10.1109 /TGRS.2008.2002030.

— ment Simulator Suite for Atmospheric Remote Sensing from spaceborne platform (ISSARS). Earth Science Technology Forum, Pasadena, CA, http://hdl .handle.net/2014/43434.

\section{NEW FROM AMS BOOKS!}

\section{Climate in the Age of Empire: Weather Observers in Colonial Canada Victoria C. Slonosky}

Weather observers have been paying close attention to the climate for centuries, at points even hoping human activity would bring change. This book shows how and why the colonialera scientific community amassed a remarkable body of detailed knowledge about Canada's climate and its fluctuations. Covering work by early French and British observers, the book presents excerpts from weather diaries and other records that, more than the climate itself, reveal colonial attitudes toward it.

Victoria C. Slonosky studied climatology at McGill University and the Climatic Research Unit in the UK 